\title{
Avoiding bad complement in Alzheimer's disease
}

Despite evidence of an involvement of inflammatory and immunological mechanisms in the pathogenesis of Alzheimer's disease, the therapeutic benefits of anti-inflammatory agents are still unclear. A study published in the Journal of Immunology shows for the first time that inhibition of the receptor for the pro-inflammatory complement factor $\mathrm{C} 5 \mathrm{a}(\mathrm{C} 5 \mathrm{aR})$ reduces the neuropathology and rescues cognitive deficits in two mouse models of the disease.

Alzheimer's disease is characterized by the presence of neurofibrillary tangles and amyloid- $\beta(\mathrm{A} \beta)$ plaques, which co-localize with various inflammation-related proteins and activated microglia. However, the contribution of each of these

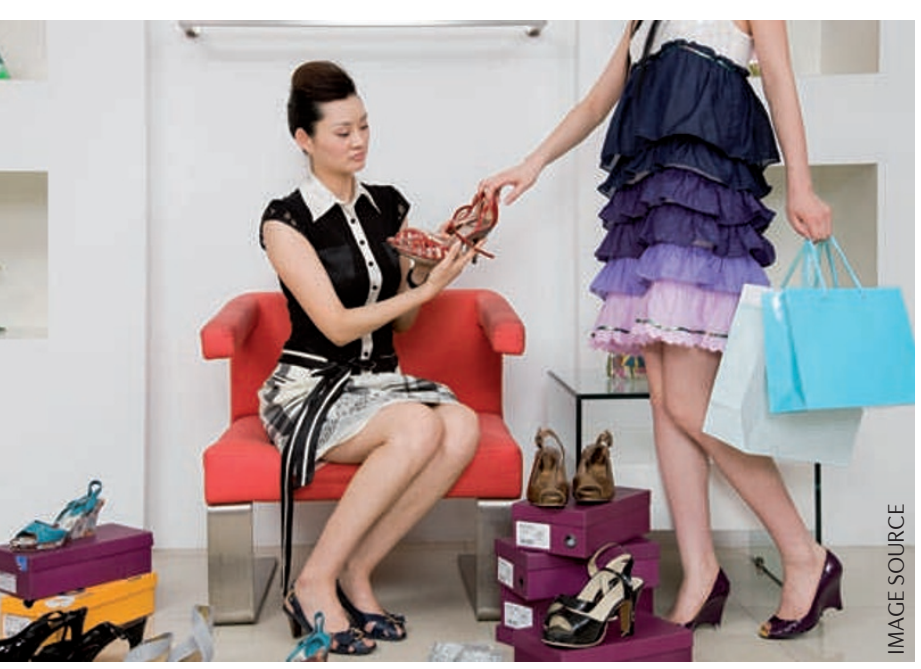

factors to cognitive decline is unclear; their presence is not sufficient for clinical diagnoses.

$\mathrm{A} \beta$ plaques have been shown to activate the complement system which, through activation factors (such as $\mathrm{C} 3 \mathrm{a}$ and $\mathrm{C} 5 \mathrm{a}$ ), triggers the recruitment and activation of phagocytes, including microglia, to sites of injury or infection. Studies on the role of activated microglia in neurodegeneration have shown conflicting results: some studies indicate that activated microglia contribute to neurodegeneration whereas others indicate that they exert a neuroprotective effect.

Here, the authors show that a small-molecule inhibitor of C5aR, PMX205, has beneficial effects in mouse models of Alzheimer's disease. Oral delivery of the compound at 10-20 $\mu$ g per ml over 12 weeks to Tg2576 mice between 12 and 15 months of age (the time frame in which there is a rapid accumulation of amyloid deposits) decreased amyloid deposits and glial cell reactivity in the hippocampus and cortex, compared with untreated animals. At these concentrations, no effect on the rate of fibril formation or toxicity was observed. Furthermore, in the 3XTg mouse model, the authors found a significant decrease in the levels of hyperphosphorylated tau, which is another hallmark of Alzheimer's disease.
Importantly, treatment with PMX205 improved the cognitive function of transgenic mice, as assessed in a passive avoidance task for contextual memory. Typically, Tg2576 mice exhibit a shorter delay in entering a dark chamber in which they have previously experienced a mild electric shock than wild-type controls. However, this behaviour was largely abolished in the PMX205treated transgenic mice, suggesting an improvement in their ability to recall the aversive stimulus.

The authors suggest that by targeting downstream events in the complement cascade it might be possible to avoid inhibition of any potential neuroprotective effects of complement activation. Given that the effects described were observed in the mice at an age when the cognitive deficits and pathological hallmarks of the disease are present, and that the parent compound of PMX205 (PMX53) has successfully passed Phase I clinical trials for inflammatory disorders, the therapeutic benefits of inhibiting complement-mediated inflammation in patients with Alzheimer's disease warrant further investigation.

Monica Hoyos Flight

ORIGINAL RESEARCH PAPER Fonseca, M. I. et al. Treatment with a $\mathrm{C} 5 \mathrm{aR}$ antagonist decreases pathology and enhances behavioral performance in murine models of Alzheimer's disease J. Immunol. 183, 1375-1383 (2009) 